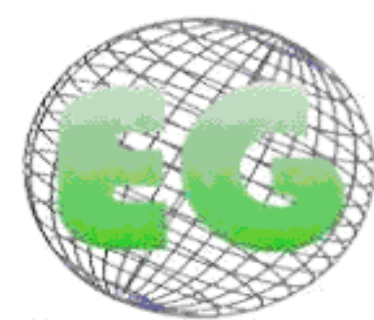

ISSN 1695-6141 $N^{\circ} 22$
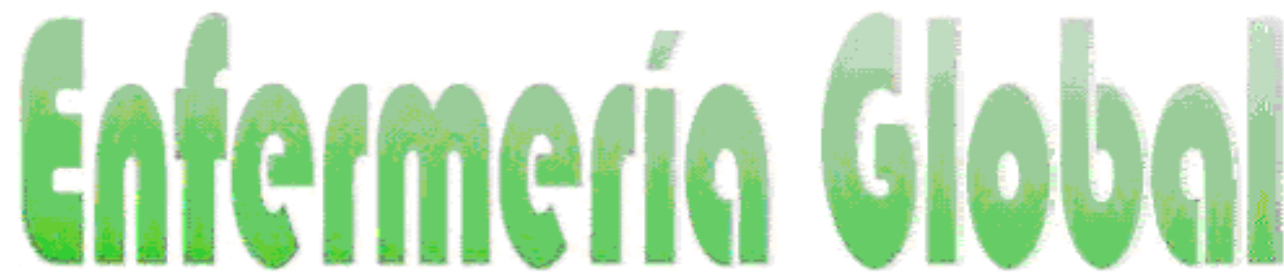

Revista electrónica trimestral de Enfermería

Abril 2011

\title{
EVALUACIÓN CLÍNICA Y HOJA DE REGISTRO DE CUIDADOS DE ENFERMERÍA DEL SISTEMA DE CONTROL FECAL EN PACIENTES CRÍTICOS
}

FECAL MANAGEMENT SYSTEM IN CRITICAL PATIENTS: CLINICAL EVALUATION AND NURSING RECORD.

\author{
*Sánchez López, MI., "Carmona Simarro, JV., **Gallego López, JM., "*Álvarez \\ Ordiales, A.
}

${ }^{*}$ DUE. Prof. Titular. Universidad CEU Cardenal Herrera. Moncada. Valencia. ${ }^{* *}$ Enfermero. Unidad de Reanimación. Hospital Clínico Universitario. Valencia.

\begin{abstract}
Palabras clave: Cuidados críticos; Diarrea; Enfermería; Flexi-seal@; Incontinencia fecal. Keywords: Critical care; Diarrhea; Nursisng; Flexi-seal $($; Fecal incontinence.
\end{abstract}

\section{RESUMEN}

La incontinencia fecal en los Pacientes Críticos produce una serie de complicaciones como son: el deterioro de la integridad cutánea de la piel, infecciones, alteración en la autoestima del paciente, etc. Esta serie de problemas puede ocasionar un aumento en la media de estancia de los pacientes ingresados con repercusiones sanitarias y económicas.

La evidencia científica nos indica que los sistemas tradicionales utilizados para la incontinencia fecal producen diferentes problemas potenciales aún sin solucionar.

El sistema de control de la incontinencia fecal Flexi-Seal ${ }^{\circledR}$ FMS (Fig. $\mathbf{n}$-1) produce una reducción de estos problemas y su efectividad está basada en diferentes estudios recientes que así lo demuestran (Martínez Estadella, G. et al, 2006), (Ares Sánchez, M. et al, 2006) (Bordes, J. et al, 2007), (Pelleschi, ME, 2008).

Es necesario describir tanto la técnica de inserción como los Cuidados de Enfermería de este nuevo dispositivo de incontinencia fecal.

\section{ABSTRACT}

Fecal incontinence in critical patients predisposes them to several complications as: tissue viability problems, infections, or patients well-being effects. This kind of problems could increase average stay hospital, with economic and health system repercussions. 
The evidence shows that traditional managements of fecal incontinence have adverse outcomes which are unresolved.

The Flexi-seal ${ }^{\circledR}$ management system (FMS), reduces these complications, and its effectiveness is based on different studies (Martínez Estadella G et al, 2006), (Ares Sánchez M et al, 2006) (Bordes J et all, 2007), (Pelleschi ME, 2008).

It is necessary to describe the insertion technique, and Nursing interventions orientated to patients who need Flexi-seal ${ }^{\circledR}$ management system (FMS), in order to improve Nursing Cares.

\section{INTRODUCCIÓN}

La diarrea es un problema muy común en el medio hospitalario. No es una enfermedad, sino un síntoma. Consiste en un aumento de la frecuencia de defecación (en r/c patrón habitual del paciente) asociado a un incremento del volumen total de heces, (más de $300 \mathrm{ml} /$ día) y una mayor fluidificación de las mismas (Lewis SM et al, 2004).

Las diarreas según su etiopatogenia pueden clasificarse en:

- Diarreas secretoras, donde hay un estímulo de los mecanismos secretores del intestino, de modo que la cantidad de agua y electrólitos secretados es mayor que los absorbidos. Dentro de este tipo encontramos las diarreas producidas por infección de escherichia coli.

- Diarreas osmóticas, se producen por la presencia de sustancias osmóticamente activas en la luz intestinal, que impiden o retrasan la absorción de agua. Es común en los síndromes de malabsorción o cuando se realizan grandes ingestas de sustancias que son mal absorbidas.

- Diarreas motoras, debidas a trastornos de la motilidad intestinal, bien por una reducción del peristaltismo (que favorecería el crecimiento bacteriano y éste desencadenaría la diarrea), bien por un aumento del tránsito gastrointestinal, que implicaría una disminución del tiempo de contacto con la mucosa del intestino, por tanto menor absorción en intestino delgado, de modo que llegaría al colon más cantidad del fluido de la que puede absorber.

El control de la diarrea es una prioridad en el cuidado de pacientes críticos (en medio hospitalario) tanto para reducir el riesgo de dermatitis y lesiones perineales, como para evitar la transmisión de infecciones nosocomiales y mejorar el confort del paciente.

Hasta ahora las medidas físicas para la contención de la incontinencia fecal causada por diarrea eran, entre otras, el uso de pañales, empapadores de celulosa y sondas rectales semirrígidas. Todas estas medidas no permiten un control adecuado de la incontinencia, ni evitan muchas de sus complicaciones. De ahí la ventaja que supone el uso de dispositivos de control fecal, como el Flexi-Seal $B_{\text {FMS. }}$ 
Figura 1. Sistema de control de la incontinencia fecal Flexi-Sea ${ }^{\circledR}$ FMS.

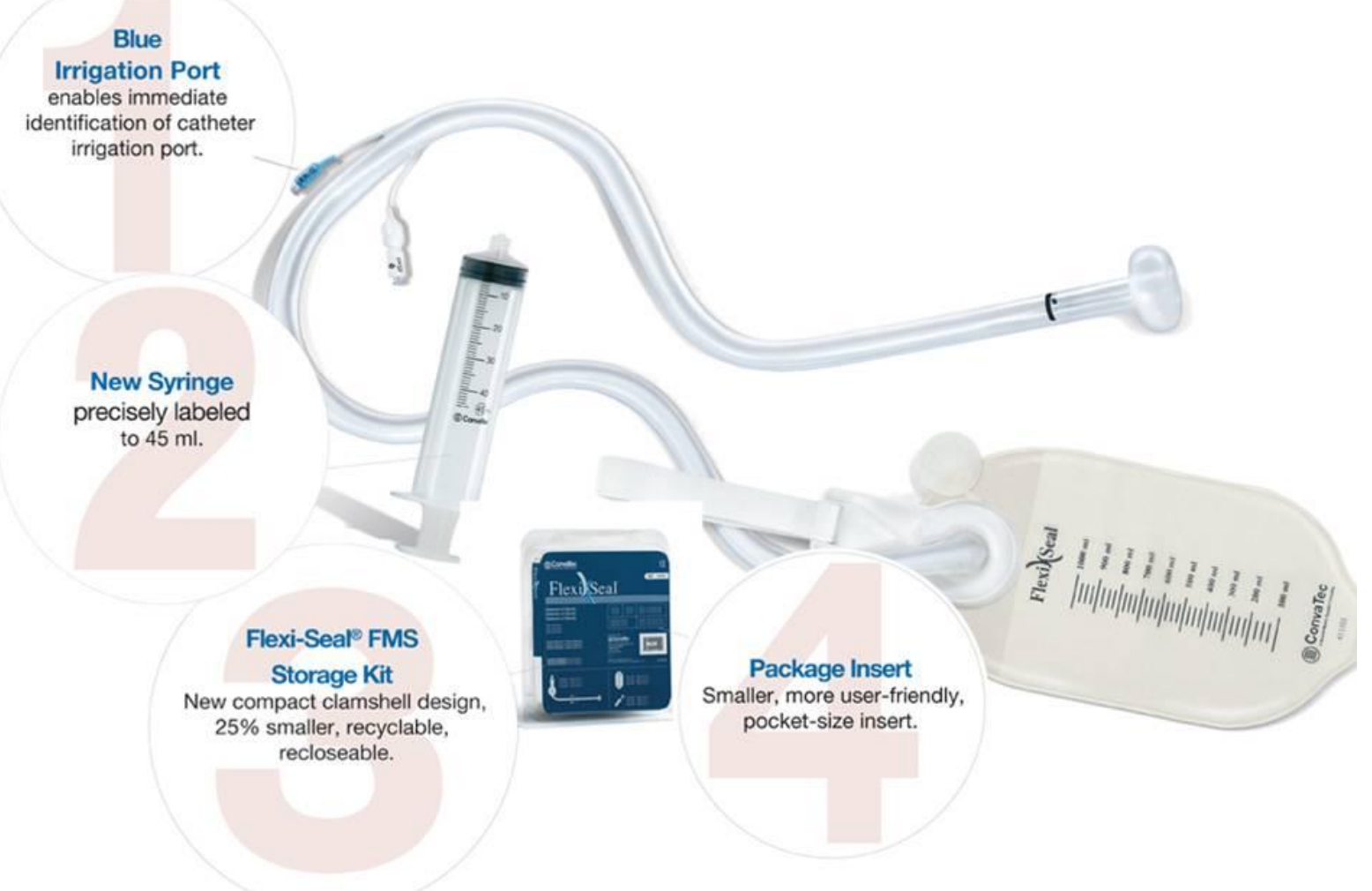

\section{SISTEMA DE CONTROL FECAL FLEXI-SEAL ${ }^{\circledR}$ FMS}

\section{Indicaciones}

- Pacientes conscientes con deposiciones líquidas o semilíquidas, encamados y con incontinencia fecal.

- Pacientes inconscientes con deposiciones líquidas o semilíquidas.

- Pacientes con limitación de la movilidad y diarrea.

- Pacientes con lesiones graves perineales.

El dispositivo no deberá permanecer colocado más de 29 días. (Ref. Fabricante)

\section{Contraindicaciones}

- Pacientes tras cirugía intestinal distal: recto, sigma, etc.

- Pacientes con tumoraciones en recto.

- Pacientes con estenosis rectal.

No se recomienda en pacientes menores de 18 años. (Ref. Fabricante) 


\section{Elementos}

El sistema de control fecal Flexi-Seal ${ }^{\circledR}$ FMS se compone de los siguientes elementos:

- Un catéter de silicona, con globo de retención de baja presión en su extremo distal.

Figura 2. Detalle del globo de silicona de baja presión.

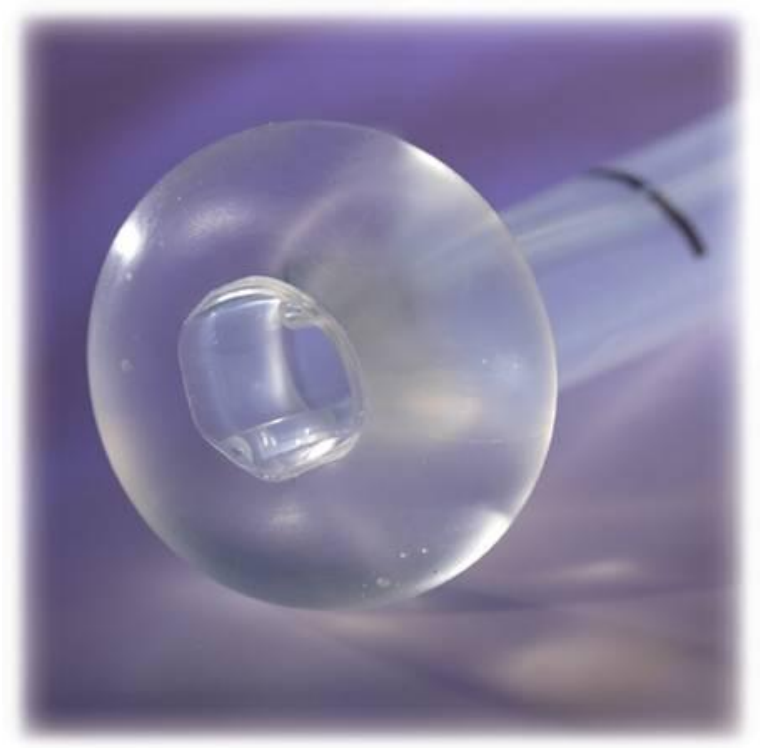

- Puerto para llenado del balón (se aconseja utilizar agua bidestilada) con señal indicadora de inflación. El volumen de llenado será de $45 \mathrm{ml}$ (leyenda impresa "45 ml'). La jeringa está marcada hasta $45 \mathrm{ml}$. Esto reduce el riesgo de error en el llenado del globo.

Figura 3. Puerto para llenado del balón.

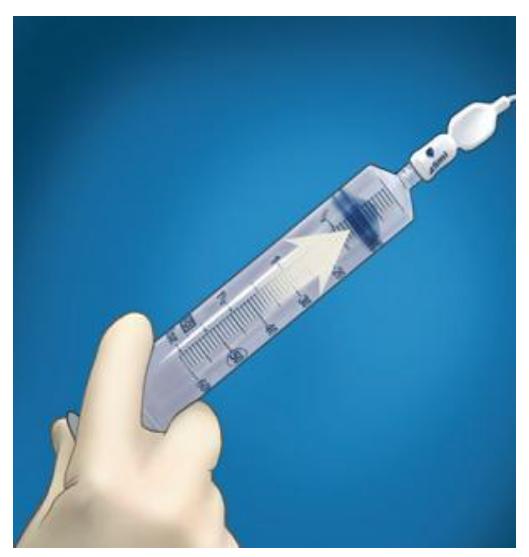

- Puerto de irrigación del sistema para realizar lavados (leyenda impresa "IRRIG").

- Bolsa recolectora (con filtro de carbón) transparente y cerrada con sujeción y escala de medición de 100 a $1000 \mathrm{ml}$. 
Figura 4. Bolsa recolectora transparente y cerrada con sujeción.

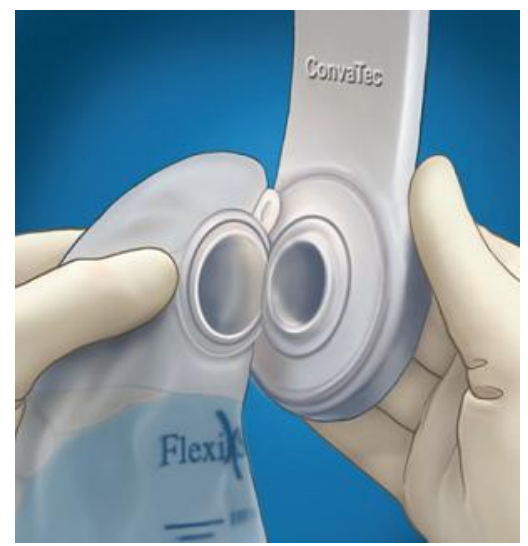

\section{Colocación del sistema}

- Realizar una inspección visual y tacto rectal en busca de contraindicaciones de la inserción de la sonda: hemorroides, estenosis, úlceras, fecalomas, etc.

- Si el paciente está consciente, explicarle el procedimiento que vamos a realizar y pedir su colaboración.

- Comprobar la integridad del globo. Llenar la jeringa con $45 \mathrm{ml}$ de agua bidestilada y comprobar que el globo está íntegro. Retirar el volumen y dejarlo conectado al dispositivo. En numerosos estudios se ha demostrado que el llenado de dispositivos de este tipo con cloruro sódico al $0.9 \%$ (por ejemplo en la sonda uretral) puede cristalizar dentro de él, por tanto, se recomienda llenarlo con agua sin soluto para mayor seguridad (p.e. agua bidestilada).

- Colocar la bolsa colectora al dispositivo.

\section{Inserción del dispositivo}

- Algunas referencias bibliográficas citan la inserción del dispositivo bajo sedación (Bordes, J. et al, 2007).

- Colocar al paciente en decúbito lateral izquierdo, con las piernas flexionadas hacia el tórax.

- Desplegar el dispositivo a lo largo de la cama.

- Una vez colocados los guantes, se inserta el dedo índice, previamente lubricado (se aconseja lubrificantes "al agua") dentro de la hendidura del globo (diseñada para ese fin), que deberá también ser lubrificado). La experiencia nos indica que la colocación del dispositivo sin lubrificar previamente el dedo a introducir, provoca el arrastre accidental del mismo. Los lubrificantes al agua facilitan mejor la introducción del catéter frente a otros. 
Figura 5. Inserción del dispositivo (dedo índice, previamente lubricado).

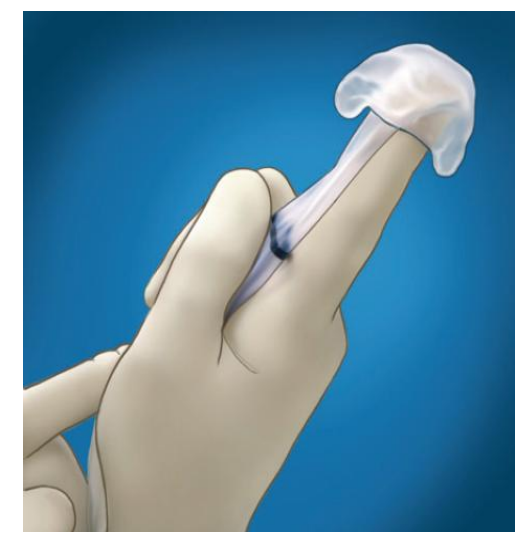

- Si el paciente está consciente, le explicaremos que realice la maniobra de Valsalva y deje de hacerla, relajándose en ese momento, que aprovecharemos para introducirle el dispositivo.

- Insertar el globo a través del esfínter anal hasta llegar a la ampolla rectal).

Figura 6. Inserción del globo a través del esfínter anal hasta llegar a la ampolla rectal.

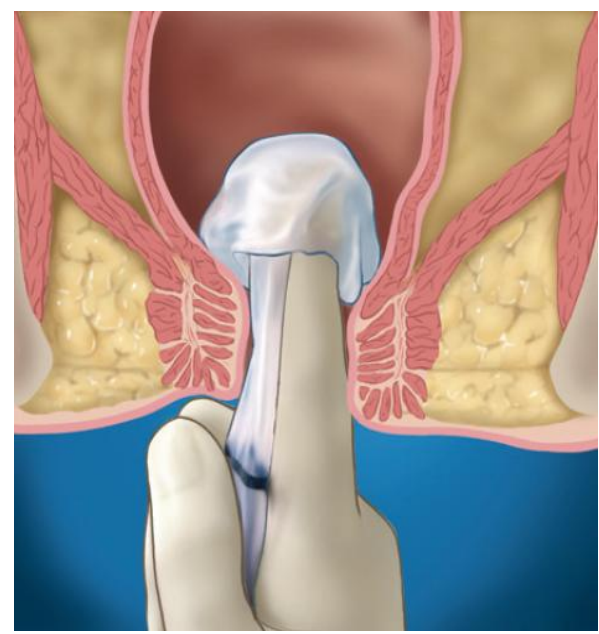

- Llenar lentamente el globo con $45 \mathrm{ml}$ de agua bidestilada. La cámara oval situada a continuación de la conexión de llenado del globo indicará el "inflado" correcto. Una cámara demasiado expandida indica una colación incorrecta del sistema, fuera de la ampolla rectal. En este caso, vaciar el globo en su totalidad y colocarlo en su lugar correcto.

- Tirar suavemente del sistema para comprobar su anclaje. 
Figura 7. Llenado del globo.

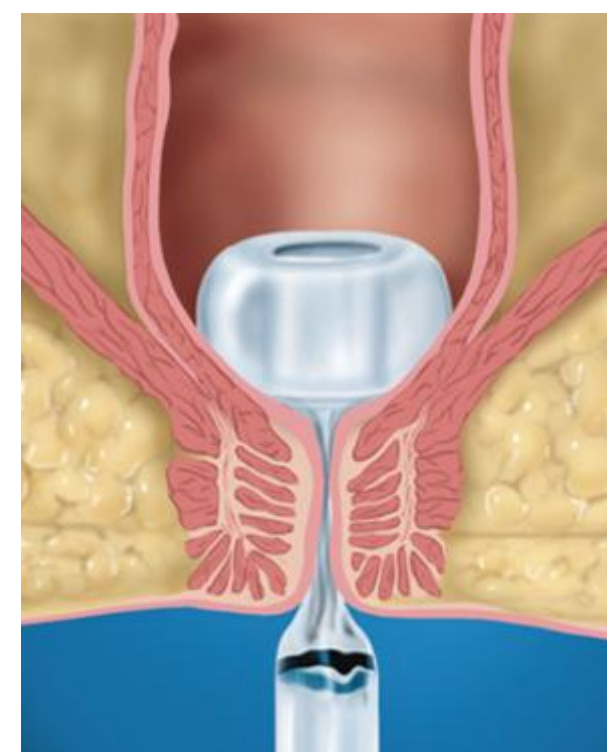

- Colocar la bolsa recolectora "en declive" para facilitar la salida de las heces.

- Fijar la bolsa recolectora a la cama, de manera que el dispositivo no quede demasiado tirante.

\section{Mantenimiento del dispositivo y cuidados sobre el paciente}

- Comprobar que el dispositivo no esté acodado (un acodamiento prolongado podría ocasionar una obstrucción del mismo).

- Comprobar que el dispositivo no esté tirante, evitando una retirada accidental.

- Mantener la zona anal y peri-dispositivo, limpia y seca.

- Valorar el volumen y consistencia de las heces.

- A valorar lavados sistemáticos por día o turno, según estado de las heces.

- Vaciar la bolsa recolectora al menos una vez al día y siempre que sea necesario.

- Registrar los procedimientos y cuidados en la hoja de enfermería.

\section{Retirada del dispositivo}

El dispositivo se retirará ante una disminución del débito y frecuencia de deposiciones, ante la presencia de heces "normales", dolor rectal en la zona de fijación del globo, hemorragia rectal y distensión abdominal. En cualquier caso, el dispositivo se retirará pasados 29 días (ref. fabricante).

- Extraer el agua bidestilada del globo de retención.

- Sujetar el dispositivo lo más cerca del paciente y retirarlo.

- Lavar y limpiar la zona perianal del paciente.

- Documentar en la hoja de registro.

\section{OBJETIVOS}

La creación de una hoja de registro y valoración enfermera del dispositivo de control fecal Flexi-Seal $®$ FMS para realizar un "trabajo de investigación" que permita mejorar los 
cuidados de Enfermería en estos pacientes, y valorar los beneficios del uso del dispositivo a varios niveles.

\section{MATERIAL Y MÉTODOS}

- Revisión bibliográfica de publicaciones relacionados con la incontinencia fecal, diarrea, medidas de control y cuidados aplicados a estos pacientes, y dispositivo Flexi-Seal ® FMS.

- Creación de una hoja de registro y valoración enfermera del dispositivo Flexi-Seal ® FMS.

- Realización de un sondeo para valorar la validez de la hoja de registro.

\section{RESULTADOS}

- Tras realizar la búsqueda bibliográfica, hemos observado la escasa bibliografía relacionada con el producto a estudio y cuidados relacionados.

- Dado que la aplicación de la hoja de registro se ha implementado en un sondeo, no es posible extraer resultados generalizables.

- Diferentes estudios muestran cómo los métodos tradicionales de contención de la incontinencia fecal acarrean diversas complicaciones.

\section{CONCLUSIONES / DISCUSIÓN}

- Es necesario realizar una investigación más profunda sobre la utilización del dispositivo Flexi-Seal @ FMS, para determinar su efectividad a varios niveles; disminución en la aparición de lesiones perineales, disminución en la transmisión de infecciones, y reducción de gasto económico.

- La hoja de registro va a suponer una mejora en los cuidados de pacientes a estudio, y aumento de bienestar.

- Nos proponemos como objetivo fundamental la realización de un trabajo de investigación multicéntrico con una muestra representativa que permita obtener resultados generalizables.

\begin{tabular}{|c|c|c|}
\hline \multicolumn{3}{|c|}{$\begin{array}{c}\text { Sistema de control fecal Flexi-Seal }{ }^{\circledR} \text { FMS } \\
\text { Registro de enfermería }\end{array}$} \\
\hline \multicolumn{2}{|l|}{$\begin{array}{l}\text { Nombre y apellidos: } \\
\text { Diagnóstico: } \\
\text { Edad: }\end{array}$} & $\begin{array}{l}\text { № Ha Clínica: } \\
\text { Fecha: } \\
\text { Sexo: }\end{array}$ \\
\hline \multicolumn{3}{|c|}{ ANTES DE LA COLOCACIÓN } \\
\hline \multirow[t]{2}{*}{ Estado de la piel } & \begin{tabular}{|l} 
UPP \\
Sí $\square$ No $\square$
\end{tabular} & $\begin{array}{l}\text { Grado } \\
1 \square \\
2 \square \\
3 \square \\
4 \square\end{array}$ \\
\hline & \multicolumn{2}{|c|}{\begin{tabular}{|l} 
Localización: \\
\end{tabular}} \\
\hline ¿Ha comprobado la i & d del balón & Sí $\square$ No $\square$ \\
\hline
\end{tabular}




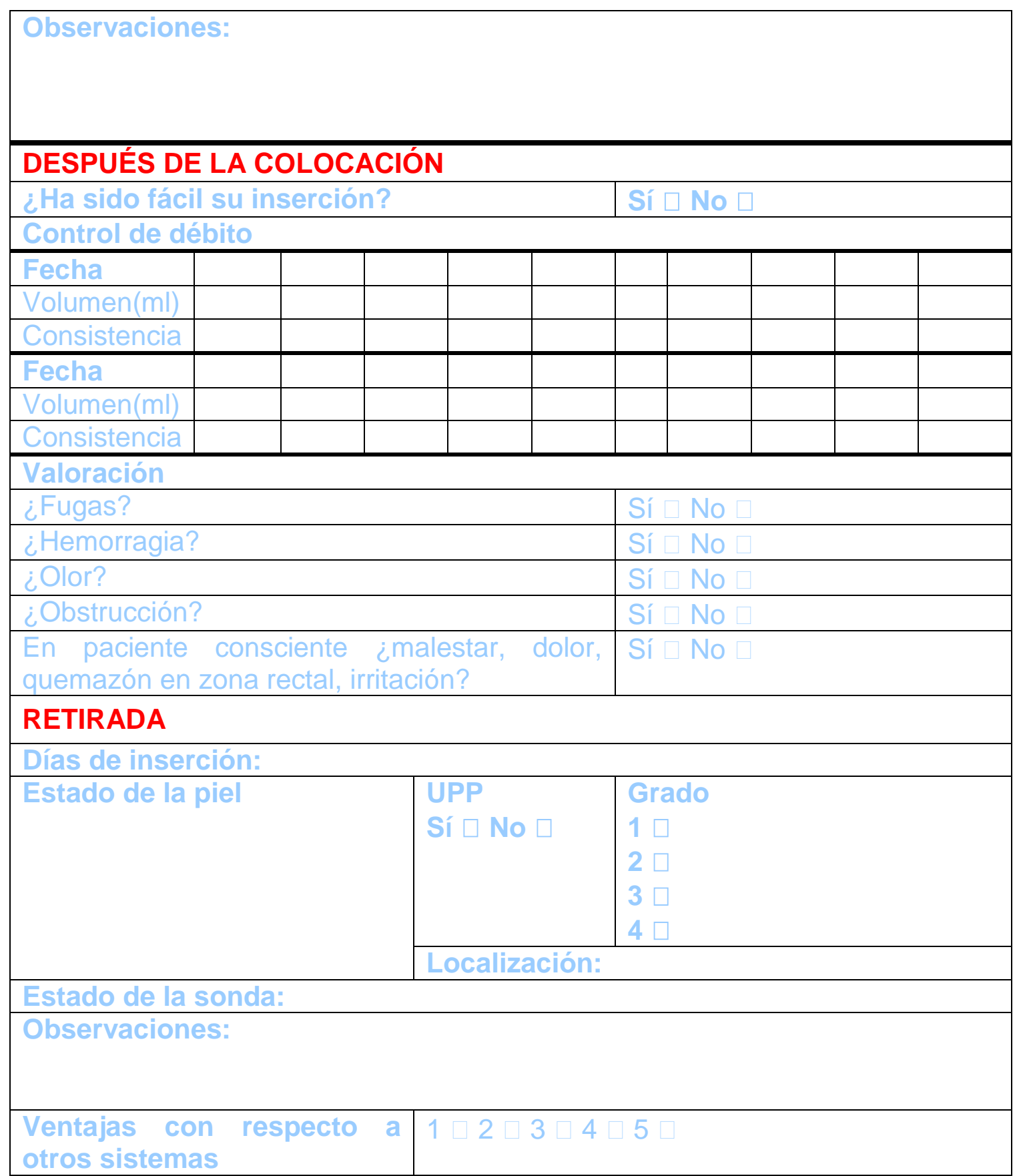

\section{BIBLIOGRAFÍA}

1. Ares Sánchez M, Santos Moraga G. (2006). Utilización de un dispositivo para el manejo de la incontinencia fecal en el paciente quemado crítico. II Congreso Nacional de Enfermería de Quemados y Cirugía Plástica. Málaga.

2. Bordes J, Goutorbe P, Asensio Y, Meaudre E, Dantzer E. (2007). A non-surgical device for faecal diversion in the management of perineal burns. Elsevier.

3. Burns. (2004). Investigación en Enfermería. 3aㅡ edición. Elsevier España. 
4. Convatec SL. Flexi-Seal ${ }^{\circledR}$. Instrucciones de uso Convatec SL. A Bristol-Myers Squibb Company. www.convatec.es

5. Gordon M. (2003). Manual de diagnósticos enfermeros. 10ª edición. Elsevier. Mosby.

6. Lewis SM. (2004). Enfermería Médico Quirúrgica. Valoración y Cuidados de Problemas Clínicos. Elsevier. Barcelona.

7. Martínez Estadella G, Baulés Conesa MJ, Rausa Braguklat E, Izquierdo Fuentes T, Vía Clavero G, Fernández Martín MJ. (2006). Manejo de la incontinencia fecal en el paciente crítico mediante la utilización de un sistema avanzado de derivación. XXXII Congreso Nacional de la SEEIUC. Pamplona.

8. Padmanabhan A, Stern M, Wishin J, Mangino M, Richey K, DeSane M. (2007). Clinical Evaluation of Flexible Incontinente Management System. Am J Crit. 16:384-393.

9. Page B, Boyce A, Deans C. Camilleri-Brennan MD. (2008). Significant Rectal Bleeding as a Complication of a Fecal Collecting Device : Report of a Case. ASCRS.51:1427-1429.

10.Pelleschi ME. (2008). Clostridium difficile Associated Disease. Diagnosis Prevention, Treatment, and Nursing Care. Critical Care Nurse. 28 (1): 27-35.

11. Rozman C. (2000). Medicina Interna Farreras/Rozman. 14를 Edición. 